\title{
A CONFIDENCE ELLIPSOID APPROACH FOR MEASUREMENT COST MINIMIZATION UNDER GAUSSIAN NOISE
}

\author{
Berkan Dulek and Sinan Gezici \\ Department of Electrical and Electronics Engineering \\ Bilkent University, Ankara, 06800, Turkey \\ \{dulek,gezici\}@ee.bilkent.edu.tr
}

\begin{abstract}
The well-known problem of estimating an unknown deterministic parameter vector over a linear system subject to additive Gaussian noise is studied from the perspective of minimizing total sensor measurement cost under a constraint on the log volume of the estimation error confidence ellipsoid. A convex optimization problem is formulated for the general case, and a closed form solution is provided when the system matrix is invertible. Furthermore, effects of system matrix uncertainty are discussed by employing a specific but nevertheless practical uncertainty model. Numerical examples are presented to discuss the theoretical results in detail.
\end{abstract}

Index Terms- Wireless sensor networks, parameter estimation, Gaussian noise, measurement cost.

\section{INTRODUCTION AND MOTIVATION}

Although the statistical estimation problem in the presence of Gaussian noise is by far the most widely known and wellstudied subject of estimation theory [1], approaches that consider the estimation performance jointly with systemresource constraints have become popular in recent years due to the surge of interest in convex optimization techniques. Distributed detection and estimation problems are the first to incorporate bandwidth and power constraints due to data processing at the sensor nodes, and data transmission from sensor nodes to a fusion node in the context of wireless sensor networks (WSNs) [2, 3]. Important results are also obtained for the sensor selection problem under various constraints on the system cost and estimation accuracy [4]. Since then, the majority of the related studies have addressed costs arising from similar system-level limitations with a relatively weak emphasis on the measurement costs due to amplitude resolution and dynamic range of the sensing apparatus.

Not much work has been performed, to the best of our knowledge, in the context of jointly designing the measurement stage from a cost-oriented perspective while performing estimation up to a predetermined level of accuracy. If adopted, such an approach will inevitably require a general and reliable method of assessing the cost of measurements applicable to any real world phenomenon under consideration as well as an appropriate means of evaluating the best achievable estimation performance without reference to any specific estimator structure. For the fulfilment of the first requirement, a novel measurement device model is suggested in [5], where the cost of each measurement is determined by the number of amplitude levels that can reliably be distinguished. As a consequence, higher resolution (less noisy) measurements demand higher costs in accordance with the usual practice. Although the proposed model may lack in capturing the exact relationship between the cost and inner workings of any specific measurement hardware, it encompasses a sufficient amount of generality to remain useful under a multitude of circumstances. Based on this measurement model, an optimization problem is formulated in [6] in order to calculate the optimal costs of measurement devices that maximize the average Fisher information for a scalar parameter estimation problem.

In this paper, we analyze the implications of the proposed measurement device model by considering a non-random vector parameter estimation problem under a constraint on the minimum acceptable estimation accuracy assuming a linear system model in the presence of Gaussian noise. ${ }^{1}$ The main contributions of our study can be summarized as follows: We (i) formulate a convex optimization problem for the minimization of the total sensor measurement cost by employing a constraint on the maximum log volume of the estimation error confidence ellipsoid; (ii) study system matrix uncertainty by employing a specific but nevertheless practical uncertainty model; (iii) obtain a closed form solution for the proposed convex optimization problem in the case of an invertible system matrix. In addition to the items listed above, we compare the performance of the proposed optimal approach against various suboptimal cost allocation schemes, and simulate the effects of system matrix uncertainty.

The remainder of the paper is organized as follows. In Section 2, the system model is introduced, and optimal cost allocation is investigated under estimation accuracy con-

\footnotetext{
${ }^{1}$ Such linear models have a multitude of applications, e.g. channel equalization, wave propagation, compressed sensing, and Wiener filtering [1]
} 


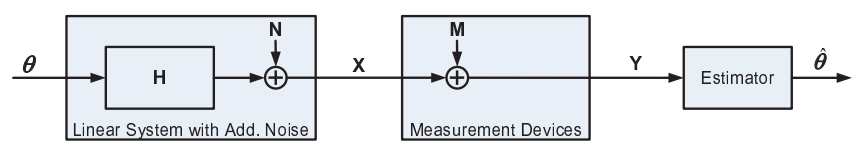

Fig. 1. Measurement and estimation systems model block diagram for a linear system with additive noise.

straints. Then, system matrix uncertainty is studied in Section 3 based on a practical uncertainty model. In Section 4, a closed form solution is obtained for the proposed optimization problem in the case of an invertible system matrix. Finally, the numerical results are presented and concluding remarks are made.

\section{OPTIMAL COST ALLOCATION UNDER ESTIMATION ACCURACY CONSTRAINT}

Consider a discrete-time system model as in Fig. 1 in which noisy measurements $\mathbf{Y} \in \mathbb{R}^{K}$ are obtained at the output of a linear system by $K$ sensors (measurement devices) and processed to estimate the value of a non-random parameter vector $\boldsymbol{\theta} \in \mathbb{R}^{L}$. The observation vector $\mathbf{X} \in \mathbb{R}^{K}$ at the output of the linear system can be represented by $\mathbf{X}=$ $\mathbf{H}^{T} \boldsymbol{\theta}+\mathbf{N}$, where $\mathbf{N} \in \mathbb{R}^{K}$ is the inherent random system noise. The system noise $\mathbf{N}$ is assumed to be Gaussian distributed with zero-mean, and independent but not necessarily identical components, i.e., $\mathbf{N} \sim \mathcal{N}\left(\mathbf{0}, \mathbf{D}_{\mathbf{N}}\right)$, where $\mathbf{D}_{\mathbf{N}}=\operatorname{diag}\left\{\sigma_{n_{1}}^{2}, \sigma_{n_{2}}^{2}, \ldots, \sigma_{n_{K}}^{2}\right\}$ is a diagonal covariance matrix. We also assume that the number of observations is at least equal to the number of estimated parameters (i.e., $K \geq L$ ) and the system matrix $\mathbf{H}$ is an $L \times K$ matrix with full row rank $L$.

Each sensor in the WSN is capable of measuring the value of a scalar physical quantity with some resolution in amplitude as $y_{i}=x_{i}+m_{i}$, where $m_{i}$ denotes the measurement noise associated with the $i^{\text {th }}$ sensor. It is reasonable to assume that measurement noise vector $\mathbf{M}$ is independent of the inherent system noise $\mathbf{N}$. In addition, the noise components introduced by sensors (the elements of $\mathbf{M}$ ) are assumed to be zero-mean independent Gaussian random variables with possibly distinct variances ${ }^{2}$, i.e., $\mathbf{M} \sim \mathcal{N}\left(\mathbf{0}, \mathbf{D}_{\mathbf{M}}\right)$, where $\mathbf{D}_{\mathbf{M}}$ is a diagonal covariance matrix given by $\mathbf{D}_{\mathbf{M}}=\operatorname{diag}\left\{\sigma_{m_{1}}^{2}, \sigma_{n_{2}}^{2}, \ldots, \sigma_{m_{K}}^{2}\right\}$.

In accordance with the model introduced in [5], the cost associated with measuring $i^{\text {th }}$ observation $x_{i}$ is given by $\mathrm{C}_{i}=$ $0.5 \log _{2}\left(1+\sigma_{x_{i}}^{2} / \sigma_{m_{i}}^{2}\right)$, where $\sigma_{x_{i}}^{2}$ denotes the variance at the input of the $i^{\text {th }}$ sensor, and $\sigma_{m_{i}}^{2}$ is the variance of the measurement noise. It is noted that a measurement device (i.e., sensor) has a higher cost if it can perform measurements

\footnotetext{
${ }^{2}$ Since Gaussian distribution maximizes the differential entropy over all distributions with the same variance, the assumption that the errors introduced by the sensors are Gaussian handles the worst-case scenario.
}

with a lower measurement variance (i.e., with higher accuracy). For an in-depth discussion on the plausibility of this measurement device model, we refer the reader to [5]. Notice that $\sigma_{x_{i}}^{2}=\sigma_{n_{i}}^{2}, \forall i \in\{1,2, \ldots, K\}$, since $\boldsymbol{\theta}$ is a deterministic parameter vector. Then, the overall cost of measuring all the components of the observation vector $\mathbf{x}$ is expressed as

$$
\mathrm{C}=\sum_{i=1}^{K} \mathrm{C}_{i}=\sum_{i=1}^{K} \frac{1}{2} \log _{2}\left(1+\frac{\sigma_{n_{i}}^{2}}{\sigma_{m_{i}}^{2}}\right)
$$

A closer look into (1) reveals that it is a nonnegative, monotonically decreasing and convex function of $\sigma_{m_{i}}^{2}, \forall \sigma_{n_{i}}^{2}>0$ and $\forall \sigma_{m_{i}}^{2}>0$. Next, we will design the optimal noise levels for the measurement devices such that the overall cost is minimized under a constraint on the minimum acceptable estimation quality.

A scalar measure of the estimation accuracy is the log volume of the $\eta$-confidence ellipsoid, which is defined as the minimum ellipsoid that contains the estimation error with probability $\eta$ [7, Sec. 7.5.2]. More explicitly,

$$
\varepsilon_{\alpha}=\left\{\mathbf{z} \in \mathbb{R}^{L} \mid \mathbf{z}^{T} \mathbf{J}(\mathbf{Y}, \boldsymbol{\theta}) \mathbf{z} \leq \alpha\right\},
$$

where $\alpha=F_{\chi_{K}^{2}}^{-1}(\eta)$ is obtained from the cumulative distribution function of a chi-squared random variable with $K$ degrees of freedom, and $\mathbf{J}(\mathbf{Y}, \boldsymbol{\theta})$ is the Fisher information matrix (FIM) of the measurement $\mathbf{Y}$ relative to the parameter vector $\boldsymbol{\theta}$. For independent Gaussian random vectors $\mathbf{N}$ and $\mathbf{M}$, it can be shown that FIM is given by [8]

$$
\mathbf{J}(\mathbf{Y}, \boldsymbol{\theta})=\mathbf{H ~ C o v}^{-1}(\mathbf{N}+\mathbf{M}) \mathbf{H}^{T}
$$

where $\operatorname{Cov}(\cdot)$ represents the covariance matrix of the random vector $\mathbf{N}+\mathbf{M}$. From independence, we have $\operatorname{Cov}(\mathbf{N}+\mathbf{M})=$ $\mathbf{D}_{\mathbf{N}}+\mathbf{D}_{\mathbf{M}}=\operatorname{diag}\left\{\sigma_{n_{1}}^{2}+\sigma_{m_{1}}^{2}, \sigma_{n_{2}}^{2}+\sigma_{m_{2}}^{2}, \ldots, \sigma_{n_{K}}^{2}+\sigma_{m_{K}}^{2}\right\}$, and $\operatorname{Cov}^{-1}(\mathbf{N}+\mathbf{M})=\operatorname{diag}\left\{1 /\left(\sigma_{n_{1}}^{2}+\sigma_{m_{1}}^{2}\right), 1 /\left(\sigma_{n_{2}}^{2}+\right.\right.$ $\left.\left.\sigma_{m_{2}}^{2}\right), \ldots, 1 /\left(\sigma_{n_{K}}^{2}+\sigma_{m_{K}}^{2}\right)\right\}$, where $\operatorname{Cov}^{-1}(\cdot)$ denotes the inverse of the covariance matrix. Then, FIM can be expressed explicitly as

$$
\mathbf{J}(\mathbf{Y}, \boldsymbol{\theta})=\sum_{i=1}^{K} \frac{1}{\sigma_{n_{i}}^{2}+\sigma_{m_{i}}^{2}} \mathbf{h}_{i} \mathbf{h}_{i}^{T}
$$

and log volume of the $\eta$-confidence ellipsoid is given as ${ }^{3}$

$$
\log \operatorname{vol}\left(\varepsilon_{\alpha}\right)=\beta-\frac{1}{2} \log \operatorname{det}\left(\sum_{i=1}^{K} \frac{1}{\sigma_{n_{i}}^{2}+\sigma_{m_{i}}^{2}} \mathbf{h}_{i} \mathbf{h}_{i}^{T}\right)
$$

where $\beta=n / 2 \log (\alpha \pi)-\log (\Gamma(n / 2+1))$, and $\Gamma(\cdot)$ denotes the Gamma function [4]. Notice that the above expression is related to the geometric mean of the eigenvalues of the FIM. Furthermore, for linear models in the form of Fig. 1

\footnotetext{
${ }^{3}$ We use 'log' without a subscript to denote the natural logarithm.
} 
but with arbitrary probability distributions for $\mathbf{N}$ and $\mathbf{M}$, it is possible to obtain an upper bound on the volume of the $\eta$-confidence ellipsoid by realizing that

$$
\mathbf{J}(\mathbf{Y}, \boldsymbol{\theta})=\mathbf{H J}(\mathbf{N}+\mathbf{M}) \mathbf{H}^{T} \succeq \mathbf{H C o v}^{-1}(\mathbf{N}+\mathbf{M}) \mathbf{H}^{T},
$$

where $\mathbf{J}(\mathbf{N}+\mathbf{M})$ indicates the FIM under a translation parameter of random vector $\mathbf{N}+\mathbf{M}$, and the symbol $\succeq$ between nonnegative definite matrices above represents the inequality with respect to the positive semidefinite matrix cone $[7,8]$.

Based on this metric, we propose the following sensor measurement cost optimization problem:

$$
\begin{aligned}
& \max _{\left\{\mu_{i}\right\}_{i=1}^{K}} \frac{1}{2} \sum_{i=1}^{K} \log _{2}\left(1-\sigma_{n_{i}}^{2} \mu_{i}\right) \\
& \text { subject to } \log \operatorname{det}\left(\sum_{i=1}^{K} \mu_{i} \mathbf{h}_{i} \mathbf{h}_{i}^{T}\right) \geq 2(\beta-\mathrm{S}),
\end{aligned}
$$

where $\mu_{i} \triangleq 1 /\left(\sigma_{n_{i}}^{2}+\sigma_{m_{i}}^{2}\right)$, and $\mathrm{S}$ is a constraint on the log volume of $\eta$-confidence ellipsoid satisfying

$$
\mathrm{S}>\beta-0.5 \log \operatorname{det}\left(\sum_{i=1}^{K} \frac{1}{\sigma_{n_{i}}^{2}} \mathbf{h}_{i} \mathbf{h}_{i}^{T}\right) .
$$

It is noted that the objective function is smooth and concave for $\forall \mu_{i} \in\left[0,1 / \sigma_{n_{i}}^{2}\right)$. Since $\log \operatorname{det}\left(\sum_{i=1}^{K} \mu_{i} \mathbf{h}_{i} \mathbf{h}_{i}^{T}\right)$ is a smooth concave function of $\mu_{i}$ for $\mu_{i} \geq 0$, the resulting optimization problem is convex [7, Sec. 3.1.5]. Consequently, it can be efficiently solved in polynomial time using interior point methods, and the numerical convergence is assured.

By introducing a lower triangular non-singular matrix $\mathbf{L}$ and utilizing the Cholesky decomposition of positive definite matrices, it is possible to rewrite the constraint using linear matrix inequalities (LMIs). To that aim, let $\sum_{i=1}^{K} \mu_{i} \mathbf{h}_{i} \mathbf{h}_{i}^{T} \succeq$ $\mathbf{L} \mathbf{L}^{T}$. Then, optimization in (6) can be expressed equivalently

$$
\begin{aligned}
\max _{\mathbf{L} \in \mathbf{U}_{L},\left\{\mu_{i}\right\}_{i=1}^{K}} & \frac{1}{2} \sum_{i=1}^{K} \log _{2}\left(1-\sigma_{n_{i}}^{2} \mu_{i}\right) \\
\text { subject to } & {\left[\begin{array}{cc}
\mathbf{I} & \mathbf{L}^{T} \\
\mathbf{L} & \sum_{i=1}^{K} \mu_{i} \mathbf{h}_{i} \mathbf{h}_{i}^{T}
\end{array}\right] \succeq 0, } \\
& \sum_{i=1}^{L} \log \mathbf{L}_{i, i} \geq(\beta-\mathrm{S}),
\end{aligned}
$$

where $\mathbf{U}_{L}$ denotes the set of lower triangular non-singular $L \times L$ square matrices, $\mathbf{L}_{i, i}$ represents the $i^{\text {th }}$ diagonal coefficient of $\mathbf{L}$, and $L$ is the dimension of $\mathbf{L}$.

\section{SYSTEM MATRIX UNCERTAINTY}

In practice, it is usually the case that there exists some uncertainty concerning the elements in the system matrix $\mathbf{H}$ [4]. Suppose that the system matrix $\mathbf{H}$ can take values from a given set $\mathcal{H}$. When the set $\mathcal{H}$ is finite, the problem can be solved using standard arguments from convex optimization [7]. However, the set $\mathcal{H}$ is in general not finite, and the solutions of such optimization problems require techniques from semi-infinite convex optimization [7]. In the following, a specific yet practically sound uncertainty model is considered. Let $\mathbf{H} \in \mathcal{H}=\left\{\overline{\mathbf{H}}+\boldsymbol{\Delta}:\left\|\boldsymbol{\Delta}^{T}\right\|_{2} \leq \epsilon\right\}$, where $\|\cdot\|_{2}$ denotes the spectral norm (i.e., the square root of the largest eigenvalue of the positive semidefinite matrix $\Delta \Delta^{T}$ ). It is possible to express this constraint as an LMI, $\Delta \boldsymbol{\Delta}^{T} \preceq \epsilon^{2} \mathbf{I}$. Suppose also that $\boldsymbol{\mu}$ is defined as the diagonal matrix $\boldsymbol{\mu} \triangleq$ $\operatorname{diag}\left\{\mu_{1}, \mu_{2}, \ldots, \mu_{K}\right\}$, and $\mathbf{W} \triangleq \mathbf{L L}^{T}$ is a symmetric positive definite matrix. Then, the constraint in (8) can be expressed in terms of $\overline{\mathbf{H}}$ and $\boldsymbol{\Delta}$ as

$$
\mathbf{W} \preceq \overline{\mathbf{H}} \boldsymbol{\mu} \overline{\mathbf{H}}^{T}+\overline{\mathbf{H}} \boldsymbol{\mu} \boldsymbol{\Delta}^{T}+\boldsymbol{\Delta} \boldsymbol{\mu} \overline{\mathbf{H}}^{T}+\boldsymbol{\Delta} \boldsymbol{\mu} \boldsymbol{\Delta}^{T},
$$

for all $\boldsymbol{\Delta} \boldsymbol{\Delta}^{T} \preceq \epsilon^{2} \mathbf{I}$. In [9, Theorem 3.3], a necessary and sufficient condition is derived for quadratic matrix inequalities in the form of (9) to be true. In the light of this theorem, (9) holds if and only if there exists $t \geq 0$ such that

$$
\left[\begin{array}{cc}
\overline{\mathbf{H}} \boldsymbol{\mu} \overline{\mathbf{H}}^{T}-\mathbf{W}-t \mathbf{I} & \overline{\mathbf{H}} \boldsymbol{\mu} \\
\boldsymbol{\mu} \overline{\mathbf{H}}^{T} & \boldsymbol{\mu}+\frac{t}{\epsilon^{2}} \mathbf{I}
\end{array}\right] \succeq 0 .
$$

Notice that (10) is both linear in $\boldsymbol{\mu}, \mathbf{W}$ and $t$. Hence, under this specific uncertainty model, we can express the optimization problem in (8) as

$$
\begin{aligned}
\max _{t, \mathbf{W} \in \mathbf{S}_{++}^{L},\left\{\mu_{i}\right\}_{i=1}^{K}} & \frac{1}{2} \sum_{i=1}^{K} \log _{2}\left(1-\sigma_{n_{i}}^{2} \mu_{i}\right) \\
\text { subject to } & {\left[\begin{array}{cc}
\overline{\mathbf{H}} \boldsymbol{\mu} \overline{\mathbf{H}}^{T}-\mathbf{W}-t \mathbf{I} & \overline{\mathbf{H}} \boldsymbol{\mu} \\
\boldsymbol{\mu} \overline{\mathbf{H}}^{T} & \boldsymbol{\mu}+\frac{t}{\epsilon^{2}} \mathbf{I}
\end{array}\right] \succeq 0 } \\
& \log \operatorname{det}(\mathbf{W}) \geq 2(\beta-\mathrm{S}), \\
& t \geq 0,
\end{aligned}
$$

and $\mathbf{S}_{++}^{L}$ denotes symmetric positive-definite $L \times L$ matrices.

\section{SPECIAL CASE - INVERTIBLE SYSTEM MATRIX}

When the system matrix $\mathbf{H}$ is an invertible matrix, it is possible to obtain the solution of the optimization problem stated in (6) in closed-form. Namely, we have

$$
\begin{aligned}
\log \operatorname{det}\left(\mathbf{H C o v}^{-1}(\mathbf{N}+\mathbf{M}) \mathbf{H}^{T}\right) & =2 \log |\operatorname{det} \mathbf{H}| \\
& -\sum_{i=1}^{K} \log \left(\sigma_{n_{i}}^{2}+\sigma_{m_{i}}^{2}\right) .
\end{aligned}
$$

Since the system matrix $\mathbf{H}$ is known, let $\alpha \triangleq \log |\operatorname{det} \mathbf{H}|$. Under these conditions, the optimization problem in (6) can 
be stated as

$$
\begin{aligned}
& \min _{\left\{\sigma_{m_{i}}^{2}\right\}_{i=1}^{K}} \frac{1}{2} \sum_{i=1}^{K} \log _{2}\left(1+\frac{\sigma_{n_{i}}^{2}}{\sigma_{m_{i}}^{2}}\right) \\
& \text { subject to } \sum_{i=1}^{K} \log \left(\sigma_{n_{i}}^{2}+\sigma_{m_{i}}^{2}\right) \leq 2(\mathrm{~S}+\alpha-\beta),
\end{aligned}
$$

where $\mathrm{S}$ and $\beta$ are as defined in (6). Notice that although the objective in (12) is a convex function of $\sigma_{m_{i}}^{2}$ 's, the constraint is not a convex set. In fact, the constraint set is what is left after the convex set $\mathcal{C}=\left\{\boldsymbol{\sigma}_{\boldsymbol{m}}^{\mathbf{2}} \succeq 0: \sum_{i=1}^{K} \log \left(\sigma_{n_{i}}^{2}+\sigma_{m_{i}}^{2}\right)>\right.$ $2(\mathrm{~S}+\alpha-\beta)\}$ is subtracted from $\left\{\boldsymbol{\sigma}_{\boldsymbol{m}}^{\mathbf{2}} \succeq 0\right\}$. Since the global minimum of the unconstrained objective function is achieved for $\sigma_{\mathbf{m}}^{2}=\infty$ which is contained in set $\mathcal{C}$ and the objective function is convex, it is concluded that the minimum of the objective function has to occur at the boundary, i.e.,

$$
\sum_{i=1}^{K} \log \left(\sigma_{n_{i}}^{2}+\sigma_{m_{i}}^{2}\right)=2(\mathrm{~S}+\alpha-\beta)
$$

must be satisfied [7]. Therefore, we can take the constraint as equality in (12). This is a standard optimization problem that can be solved using Lagrange multipliers. Hence, by defining $\varrho \triangleq 2(\mathrm{~S}+\alpha-\beta)$, we can write the Lagrange functional as

$$
\begin{aligned}
J\left(\sigma_{m_{1}}^{2}, \ldots, \sigma_{m_{K}}^{2}\right) & =\frac{1}{2} \sum_{i=1}^{K} \log _{2}\left(1+\frac{\sigma_{n_{i}}^{2}}{\sigma_{m_{i}}^{2}}\right) \\
& +\lambda\left(\sum_{i=1}^{K} \log \left(\sigma_{n_{i}}^{2}+\sigma_{m_{i}}^{2}\right)-\varrho\right),
\end{aligned}
$$

and differentiating with respect to $\sigma_{m_{i}}^{2}$, we have the optimal assignment of the noise variances to the measurement devices

$$
\sigma_{m_{i}}^{2}=\left(\gamma^{1 / K}-1\right) \sigma_{n_{i}}^{2}, \quad \text { where } \quad \gamma=\frac{2^{\varrho}}{\prod_{j=1}^{K} \sigma_{n_{j}}^{2}}
$$

For consistency, the design parameter $\mathrm{S}$ should be selected as $\varrho=2(\mathrm{~S}+\alpha-\beta)>\sum_{i=1}^{K} \log \left(\sigma_{n_{i}}^{2}\right)$ since the intrinsic system noise puts a lower bound on the minimum attainable volume of the confidence ellipsoid. Finally, if the observation variances are equal; that is, $\sigma_{n_{i}}^{2}=\sigma_{n}^{2}, i=1, \ldots, K$, employing identical measurement devices for all the observations; that is, $\sigma_{m_{i}}^{2}=\sigma_{m}^{2}=\mathrm{e}^{\varrho / K}-\sigma_{n}^{2}, i=1, \ldots, K$, is the optimal strategy. The corresponding minimized total measurement cost is given by $\varrho /(2 \log 2)-(K / 2) \log _{2}\left(\mathrm{e}^{\varrho / K}-\sigma_{n}^{2}\right)$.

\section{NUMERICAL RESULTS}

In this section, we present an example that illustrates several theoretical results developed in the previous sections. A discrete-time linear system as depicted in Fig. 1 is considered where $\boldsymbol{\theta}$ is a length-20 vector containing the unknown parameters to be estimated, $\mathbf{H}$ is a $20 \times 100$ system matrix with full

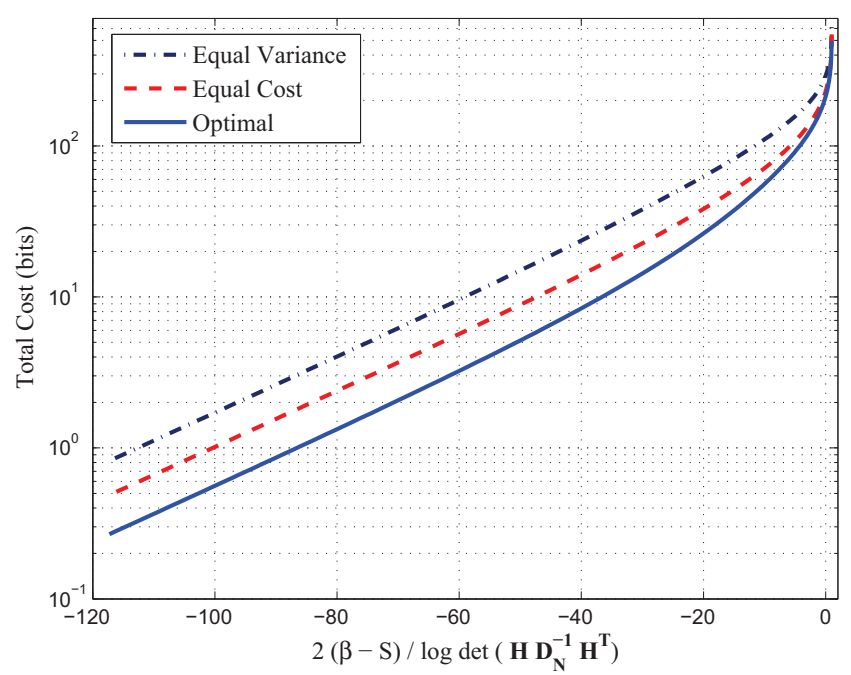

Fig. 2. Total cost versus normalized estimation accuracy constraint.

row rank, the intrinsic system noise $\mathbf{N}$ and the measurement noise $\mathbf{M}$ are length-100 Gaussian distributed random vectors with independent components. The entries of the system matrix $\mathbf{H}$ are generated from a process of independent and identically distributed uniform random variables in the interval $[-0.1,0.1]$. Also, the components of the system noise vector $\mathbf{N}$ are independently Gaussian distributed with zero mean, and it is assumed that their variances come from a uniform distribution defined in the interval $[0.05,1]$. The implication of this assumption is that the observations at the output of the linear system possess uniformly varying degrees of accuracy.

First, we investigate the cost assignment problem under perfect information on the system matrix and intrinsic noise variances. The constraint metric is expressed as the ratio of its current value to the value it attains for the limiting case when zero measurement noise variances are assumed. In addition to the optimal cost allocation scheme proposed in this paper, we also consider two suboptimal cost allocation strategies:

Equal cost to all measurement devices: In this strategy, it is assumed that a single set of measurement devices with identical costs is employed for all observations so that $\mathrm{C}_{i}=$ $\mathrm{C}, i=1,2, \ldots, K$. This, in turn, implies that the ratio of the measurement noise variance to the intrinsic system noise variance, $x \triangleq \sigma_{m_{i}}^{2} / \sigma_{n_{i}}^{2}$, is constant for all measurement devices. Then, the total cost can be expressed in terms of $x$ as $\mathrm{C}=0.5 K \log _{2}(1+1 / x)$, and similarly the FIM becomes

$$
\mathbf{J}(\mathbf{Y}, \boldsymbol{\theta})=\frac{1}{x+1} \sum_{i=1}^{K} \frac{1}{\sigma_{n_{i}}^{2}} \mathbf{h}_{i} \mathbf{h}_{i}^{T}
$$

Using this observation, the constraint function in (6) can be algebraically solved for equality to determine the value of $x$ without applying any convex optimization techniques, and the corresponding measurement variances and cost assign- 


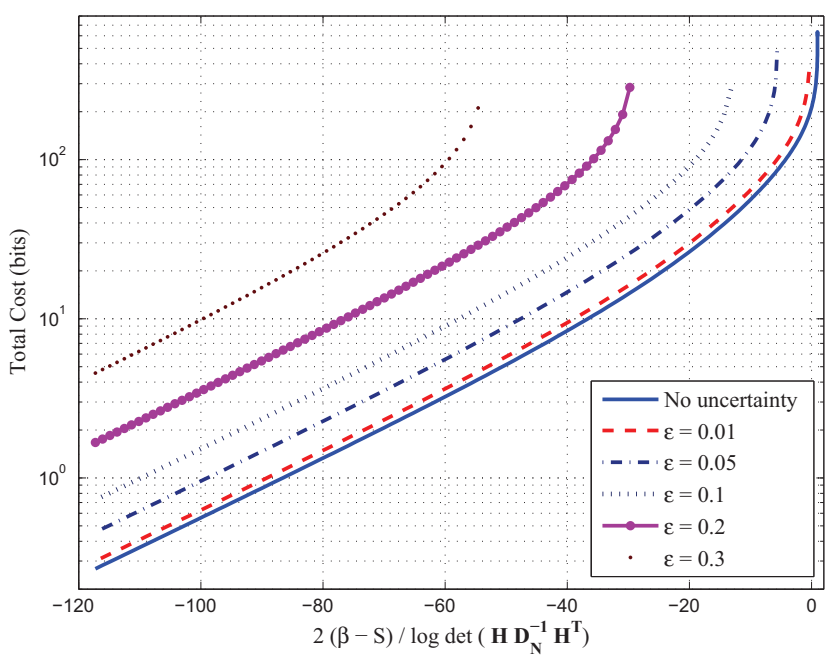

Fig. 3. Effects of system matrix uncertainty on the total measurement cost.

ments can be obtained.

Equal measurement noise variances: In this case, measurement devices are assumed to introduce random errors with equal noise variances, that is, $\sigma_{m_{i}}^{2}=\sigma_{m}^{2}, i=$ $1,2, \ldots, K$. Accordingly, $\mathrm{C}=0.5 \sum_{i=1}^{K} \log _{2}\left(1+\sigma_{n_{i}}^{2} / \sigma_{m}^{2}\right)$ and the FIM is

$$
\mathbf{J}(\mathbf{Y}, \boldsymbol{\theta})=\sum_{i=1}^{K} \frac{1}{\left(\sigma_{n_{i}}^{2}+\sigma_{m}^{2}\right)} \mathbf{h}_{i} \mathbf{h}_{i}^{T}
$$

In Fig. 2, we plot the relationship between the estimation accuracy constraint and the total measurement device cost. The performance of the optimal strategy is superior to the equal measurement device cost strategy, and the worst performance belongs to the equal measurement variance scheme. When the constraint is very restrictive (corresponding to high values of $2(\beta-S)$ ), the differences among the performances of optimal and suboptimal strategies disappear. As the constrained is relaxed, we see that the drop in the total cost maintains its pace. The performance figures are quite useful in the sense that they provide the minimum cost necessary to obtain a desired level of estimation accuracy under each strategy. Finally, in order to assign more cost to a specific observation, it is not sufficient to just know that the particular observation is reliable (i.e., has smaller variance) but we also need to know its intrinsic combinations with other observations due to the linear system matrix.

In Fig. 3, we present the results concerning the effects of system uncertainty on the optimal cost allocation problem. It is observed that the total cost increases as the amount of uncertainty in the system matrix increases for a given value of the constraint. The increase in the system matrix uncertainty also leads to smaller values of the maximum attainable estimation accuracy measures (the asymptotes where the total cost increases unboundedly).

\section{CONCLUDING REMARKS}

In this paper, a convex optimization problem has been formulated for the minimization of the total sensor measurement cost by employing a constraint on the maximum log volume of the estimation error confidence ellipsoid. Also, the system matrix uncertainty has been investigated based on a practical uncertainty model. In addition to the generic formulation, the case of an invertible system matrix has been considered, and a closed form solution has been obtained. The simulation results have been presented to compare the performance of the proposed optimal approach against various suboptimal cost allocation schemes, and to investigate the effects of system matrix uncertainty. Since linear models as in Fig. 1 have proved successful in a multitude of research areas, e.g., channel equalization, wave propagation, compressed sensing, and Wiener filtering, the results in this study can be adapted to other frameworks in addition to WSN applications.

\section{REFERENCES}

[1] S. M. Kay, Fundamentals of Statistical Signal Processing: Estimation Theory, Prentice Hall, Upper Saddle River, New Jersey, 1993.

[2] S. Appadwedula, V. V. Veeravalli, and D. L. Jones, "Energy-efficient detection in sensor networks," IEEE J. Sel. Areas Commun., vol. 23, no. 4, pp. 693-702, 2005.

[3] S. Cui, J.-J. Xiao, A. J. Goldsmith, Z.-Q. Luo, and H. V. Poor, "Estimation diversity and energy efficiency in distributed sensing," IEEE Trans. Signal Process., vol. 55, no. 9, pp. 4683-4695, Sep. 2007.

[4] S. Joshi and S. Boyd, "Sensor selection via convex optimization," IEEE Trans. Signal Process., vol. 57, no. 2, pp. 451-462, 2009.

[5] A. Ozcelikkale, H. M. Ozaktas, and E. Arikan, "Signal recovery with cost-constrained measurements," IEEE Trans. Signal Process., vol. 58, no. 7, pp. 3607-3617, 2010.

[6] B. Dulek and S. Gezici, "Average Fisher information maximisation in presence of cost-constrained measurements," Electronics Letters, vol. 47, no. 11, pp. 654-656, May 2011.

[7] S. Boyd and L. Vandenberghe, Convex Optimization, Cambridge University Press, Cambridge, UK, 2004.

[8] R. Zamir, "A proof of the Fisher information inequality via a data processing argument," IEEE Trans. Info. Theory, vol. 44, no. 3, pp. 1246-1250, May 1998.

[9] Z.-Q. Luo, J. F. Sturm, and S. Zhang, "Multivariate nonnegative quadratic mappings," SIAM J. Optim., vol. 14, pp. 1140-1162, 2002. 